\title{
Historical data on cardiovascular health in Surinamese men
}

\author{
Lizzy M. Brewster ${ }^{1 *}$ (10 and Jules Brewster ${ }^{2}$
}

\begin{abstract}
Objectives: Cardiovascular risk factor burden was recently reported to be high in the Caribbean country Suriname. However, historical data for comparison were lacking. We report here rediscovered and hitherto unpublished aggregated data of what was apparently the first population study on measured blood pressure, diabetes, and cardiovascular health in men in this country, assessed in 1973. Data had been collected to raise the awareness of the local population for cardiovascular disease risk.

Data description: We provide a table of the cardiovascular risk profile, including exercise, smoking, hypertension and diabetes of 243 Surinamese adult non-institutionalized men living in an urban setting in the capital Paramaribo in 1973. These data may help understand the cardiovascular risk factor escalation of the population in time as well as aid in projections of future cardiovascular disease in this middle income country.
\end{abstract}

Keywords: Cardiovascular risk factors, Ethnic groups, Hypertension, Diabetes, Low and middle income countries

\section{Objective}

We report historical, hitherto unpublished data that had been collected in the capital Paramaribo, Suriname in 1973, to assess the cardiovascular health status in Surinamese men, in the "Have a Heart for your Heart" (HHH) study. The HHH study aimed at raising awareness of the local population for heart disease. Women had not been included because cardiovascular risk was perceived to be considerably higher in men. At the time of the study the population size of Paramaribo was around 150,000, with a national population of 320,000 citizens of whom $65 \%$ lived in an urban setting.

These data from 1973 might aid in the understanding of the high cardiovascular risk burden recently reported in the randomly selected urban citizens participating in the Healthy Life in Suriname (HeliSur) study [1, 2]. In this study in the population of Paramaribo $(\mathrm{N}=250.000$ citizens, with a national population of 560.000), a random sample $(\mathrm{n}=1800)$ of citizens aged $18-70$ years was drawn in $2013(n=1157$ included, mean age 42 years; 37\% men;

\footnotetext{
*Correspondence: mail@lizzybrewster.net

${ }^{1}$ Creatine Kinase Foundation, Amsterdam, The Netherlands

Full list of author information is available at the end of the article
}

$40 \%$ of African ancestry and $43 \%$ of South Asian ancestry) $[1,2]$.

In the HeliSur study, around $10 \%$ of the participants reported heavy, and 7\% reported moderate regular leisure exercise (respectively 17 and $8 \%$ in men), 30\% ever smoked tobacco (55\% of the men), mean body mass index $\left(\mathrm{kg} / \mathrm{m}^{2}\right)$ was 27.8 (SEM 0.2), with $25.6(0.2)$ in men; and $40 \%$ ( $41 \%$ of the men) had blood pressure levels higher than $140 \mathrm{~mm} \mathrm{Hg}$ systolic or $90 \mathrm{~mm} \mathrm{Hg}$ diastolic or were using antihypertensive drugs. Diabetes was found in 15\% of the participants ( $13 \%$ in men), thus the risk factor burden in this urban, middle income country population was high. However, we had no previous data to assess time trends in risk factor prevalence $[1,2]$.

\section{Data description}

The aim of the HHH study in 1973 had been to raise awareness for cardiovascular risk factors and cardiovascular disease. Men aged 30 years and older visiting the 15th national Surinade trade fair in Paramaribo in October 1973 had been requested oral informed consent to participate.

Participants had received information about cardiovascular disease from trained medical staff, and had filled out a questionnaire, including questions on physical 
Table 1 Overview of data files

\begin{tabular}{llll}
\hline Label & Name of data file/data set & File types (file extension) & Data repository and identifier \\
\hline Data file 1 & $\begin{array}{c}\text { Selected cardiovascular risk factors in men, Parama- } \\
\text { ribo, Suriname }\end{array}$ & Word (.docx) & $\begin{array}{c}\text { Figshare: https://doi.org/10.6084/m9.figshare.72031 } \\
40 \text { [3] }\end{array}$ \\
\hline
\end{tabular}

exercise, smoking and self-reported diabetes. Furthermore, self-defined ethnicity was registered, and height, weight, and blood pressure had been measured. Hypertension was defined as blood pressure levels higher than $140 \mathrm{~mm} \mathrm{Hg}$ systolic or $90 \mathrm{~mm} \mathrm{Hg}$ diastolic, or using antihypertensive drugs. Participants with an abnormal cardiovascular risk profile were well informed regarding the medical care they needed and referred to the family doctor. Cardiologist Dr. J. Guda, who had supervised the data collection in 1973 is deceased. J. Brewster, one of the authors of this report, had collected the data, but these were never published. Only the recently rediscovered aggregated data are currently available (Table 1, Data file 1) $[3]$.

\section{Limitations}

The limitations of these data are the inclusion of men visiting a national trade fair with the exclusion of women, and the limited data available. The main strength is the unicity of the data, as far as we know the only source of hitherto unpublished historical population data on the high cardiovascular disease risk burden in this middle income country.

\section{Abbreviations}

$\mathrm{HHH}$ : Have a Heart for your Heart study; HeliSur: Healthy Life in Suriname study; SEM: standard error of the mean.

\section{Authors' contributions}

JB had collected the data, JB and LB performed data analysis and interpretation of the data, LB and JB drafted the paper and revised it critically for important intellectual content. Both authors read and approved the final manuscript.

\section{Author details \\ ${ }^{1}$ Creatine Kinase Foundation, Amsterdam, The Netherlands. ${ }^{2}$ Diaconessen Hospital, Paramaribo, Suriname.}

\section{Acknowledgements}

None.

\section{Competing interests}

The authors declare that they have no competing interests.

\section{Availability of data materials}

The aggregated data generated during and/or analysed during the current study can be accessed in the Figshare repository: https://doi.org/10.6084/ m9.figshare.7203140. Please see the Table and Reference list for details and links to the data.

\section{Consent for publication}

Not applicable.

\section{Ethics approval and consent to participate}

The HHH study (1973) was non-therapeutic, nonclinical population research in accord with the ethical principles of the Declaration of Helsinki of the World Medical Association (WMA) (1964). The study was considered to conform to moral and scientific principles, and to be of negligible risk with potential benefit for the participants. The study was conducted by medically qualified staff. Voluntarily given verbal informed consent to participate was obtained prior to inclusion from healthy, ambulant, autonomous (non-dependent), nonvulnerable, non-institutionalized, adult individuals who visited a trade fair. The study took place before the WMA recommended in 1975 the establishment of independent committees of research ethics to assess all medical research involving people, and before medical research and institutional review boards were regulated by law and written rather than verbal informed consent became more imperative. There was no reward, offer, or sanction in order to obtain consent, and the study entailed no intervention or withholding of intervention. The available aggregated data do not contain identifiable human data. The publication of the hitherto unpublished result data is in compliance with research ethics to maximize the benefit of the findings.

The HeliSur study (2013) was conducted according to the principles of the Declaration of Helsinki (59th WMA General Assembly, Seoul, October 2008) and in accordance with the Medical Research Involving Human Participants Act. Ethical clearance was obtained from the Ethics Committee of the Ministry of Health in Suriname in 2012 (VG021-2012). All subjects gave written informed consent to participate prior to their inclusion.

\section{Funding}

Lizzy Brewster is supported by the Creatine Kinase Foundation (CKF) Amsterdam, Project Number 18CKF905PH20502. CKF actively supported this research on cardiovascular disease by ethnicity, including the study design, data collection and analysis, and the publication in peer reviewed journals.

\section{Publisher's Note}

Springer Nature remains neutral with regard to jurisdictional claims in published maps and institutional affiliations.

Received: 20 October 2018 Accepted: 19 December 2018

Published online: 22 December 2018

\section{References}

1. Nannan Panday R, Haan Y, Diemer F, Punwasi A, Rommy C, Heerenveen I, van Montfrans GA, Brewster LM. Chronic kidney disease and kidney health care status: the healthy life in suriname (HeliSur) study. IAEM. 2018. https://doi.org/10.1007/s11739-018-1962-3.

2. Baldew SM, Diemer FS, Cornelissen V, Oehlers GP, Brewster LM, Toelsie JR, Vanhees L. Physical activity and obesity: is there a difference in association between the Asian- and African-Surinamese adult population? Ethn Health. 2017;1:1-13.

3. Brewster LM, Brewster J. Data table for historical cardiovascular health in Surinamese men. Figshare. Dataset. 2018. https://doi.org/10.6084/ m9.figshare.7203140. 